\section{G265(P) GASTROENTERITIS AGGRESSIVE VERSUS SLOW TREATMENT FOR REHYDRATION (GASTRO STUDY): A DESCRIPTIVE ANALYSIS}

\begin{abstract}
1,2K Houston, 1,2J Gibb, 'A Mpoya, ${ }^{1} \mathrm{~N}$ Obonyo, ${ }^{3} \mathrm{P}$ Olupot-Olupot, ${ }^{4} \mathrm{E}$ George, ${ }^{4} \mathrm{D}$ Gibb,
$1,{ }^{2} \mathrm{~K}$ Maitland. 'Clinical Trials, KEMRI Wellcome Trust Research Programme, Kilifi, Kenya; ${ }^{2}$ Department of Paediatrics, Faculty of Medicine, St Mary's Campus, London, UK; ${ }^{3}$ Mbale Clinical Research Institute, Mbale Clinical Research Institute, Mbale, Uganda; ${ }^{4}$ Medical Research Council (MRC) Clinical Trials Unit, University College London, London, UK
\end{abstract}

10.1136/archdischild-2018-repch.258

Aims The World Health Organisation (WHO) rehydration guidelines (Plan C) for children with acute gastroenteritis (AGE) and severe dehydration are widely practiced in resource-poor settings, yet have never been evaluated in a clinical trial. GASTRO study will compare the safety and efficacy of two rehydration regimens: standard rapid rehydration (Plan C) versus a slower regimen and inform definitions for outcomes of a larger phase III trial.

Methods GASTRO is a multi-centre, open Phase II randomised controlled trial of 120 children aged 2 months to 12 years admitted with severe dehydration secondary to AGE. Children with severe malnutrition, chronic diarrhoea and known congenital/rheumatic heart disease are excluded. Children are enrolled in 3 centres in East Africa and randomised 1:1 to standard rapid rehydration (WHO plan ' $\mathrm{C}$ ' - $100 \mathrm{mls} / \mathrm{kg}$ over 3-6 hours according to age, plus additional boluses for children presenting in shock) or to a slower rehydration regimen $(100 \mathrm{mls} / \mathrm{kg}$ given over 8 hours and without additional boluses). Primary outcome is frequency of adverse events. Secondary outcomes focus on measures related to assessment of severity of dehydration, and response to treatment.

Results Enrolment is ongoing. By 21 st September 201761 children had been enrolled. Baseline characteristics across the two groups are consistent: median age 9 months (IQR 7-14), $67 \%$ males, median duration of diarrhoea and vomiting is 3 days, the majority are lethargic, thirsty and irritable on admission $(70 \%)$ and main features of dehydration are sunken eyes (100\%), prolonged skin pinch (64\%), reduced/absent tears (100\%), dry/sticky mucous membranes (98\%). Features of shock (cool peripheries, weak and fast pulse, and prolonged capillary refill) exist in 33\% of the patients at admission, and no child had severe hypotension. Median weight loss is $5.7 \%$. We have not completed recruitment and therefore cannot present outcomes at this stage.

Discussion There have been two main challenges when operationalising this trial. Firstly staff training and confidence with ensuring accurate fluid balance documentation. Secondly there has been slow recruitment as a result of national medical strikes and a severe drought across East Africa resulting in higher numbers of children fulfilling severe malnutrition anthropometric criteria and therefore being excluded.

\section{G266(P) ADOLESCENT COHORTS ASSESSING GROWTH, CARDIOVASCULAR AND COGNITIVE OUTCOMES IN LOW AND MIDDLE-INCOME COUNTRIES}

${ }^{1} \mathrm{JL}$ Ward, ${ }^{2} \mathrm{~K}$ Harrison, ${ }^{1} \mathrm{RM}$ Viner, ${ }^{3,4} \mathrm{~A}$ Costello, ${ }^{3} \mathrm{M}$ Heys. ${ }^{1}$ Institute of Child Health, University College London, London, UK; ${ }^{2}$ Department of Paediatrics, University College London Hospitals NHS Foundation Trust, London, UK; ${ }^{3}$ Institute of Global Health, University College London, London, UK; ${ }^{4}$ Department of Maternal, Newborn, Child and Adolescent Health, World Health Organisation, Geneva, Switzerland

10.1136/archdischild-2018-rcpch.259
Life-course studies are needed to explore how exposures during adolescence, particularly puberty, contribute to later cardiovascular risk and cognitive health in low and middle-income countries (LMIC), where $90 \%$ of the world's young people live. The extent of any existing cohorts investigating these outcomes in LMIC has not previously been described.

Methods We performed a systematic literature review to identify population cohort studies of adolescents in LMIC that assessed anthropometry and any of cardiovascular risk (blood pressure, physical activity, plasma glucose/lipid profile and substance misuse), puberty (age at menarche, Tanner staging, or other form of pubertal staging) or cognitive outcomes. Studies that recruited participants on the basis of a pre-existing condition or involved less than 500 young people were excluded.

Findings 1829 studies were identified, and 24 cohorts fulfilled inclusion criteria based in Asia (10), Africa (6) and South/Central America (8). 14 (58\%) of cohorts identified were based in one of four countries; India, Brazil, Vietnam or Ethiopia. Only 2 cohorts included a comprehensive cardiovascular assessment, tanner pubertal staging, and cognitive outcomes.

Conclusion Improved utilisation of existing datasets and additional cohort studies of adolescents in LMIC that collect contemporaneous measures of growth, cognition, cardiovascular risk and pubertal development are needed to better understand how this period of the life course influences future noncommunicable disease morbidity and cognitive outcomes.

\section{G267(P) PROBLEMS OF DUAL VULNERABILITY IN NUTRITION; A QUALITATIVE STUDY OF OLDER PERSONS CARING FOR UNDER FIVE YEAR OLDS IN POST-DISASTER HAITI}

${ }^{1} \mathrm{SJ}$ Raybould, ${ }^{2} \mathrm{TL}$ Ward, ${ }^{1} \mathrm{R}$ Burnett, ${ }^{3} \mathrm{~L}$ Manikam, ${ }^{4} \mathrm{M}$ Tibe, ${ }^{1} \mathrm{~B}$ Munslow. ${ }^{1}$ Humanitarian Studies, Liverpool School of Tropical Medicine and Hygiene, Liverpool, UK: ${ }^{2}$ Barts Cancer Insititute, Barts and The London School of Medicine and Dentistry, London, UK; ${ }^{3}$ Population, Policy and Practice, UCL Great Ormond Street Institute of Child Health, London, UK; ${ }^{4}$ Medicare Haiti, Medicare Project, Port-Au Prince, Haiti

\subsection{6/archdischild-2018-rcpch.260}

Situation Malnutrition plays a significant role in under-5 mortality rates following disasters. As such there is a need for an effective humanitarian response; in keeping with the social and cultural context. Older people play an important role in childcare in non-western societies, which often increases in displacement contexts. Until now policy in general, and nutrition programmes in particular, have focused on the motherchild dyad neglecting children not cared for principally by their mother.

Aim To gather information on possible barriers to older carers accessing nutritional services/support for the children they care for and explore ways to overcome these obstacles.

Method A qualitative approach was taken which aimed to explore the experiences of the carers themselves. Focus group discussions and semi-structured interviews provided data which was analysed using grounded theory approach.

Results Data collected showed that older carers have similar needs to other caregivers in emergency settings; needs which are often amplified by age. Moreover, many barriers to accessing services were elicited, including issues with targeting and advertising of nutritional programs, alongside physical and psychosocial barriers to assessing existing support. Participants generated potential solutions to each identified barrier as well as highlighting a vital need to address overall attitudes to older carers within INGOs and other service providers. 
Conclusion As older people in Haiti were often the sole carers of under- $5 \mathrm{~s}$, there is a need to recognise their importance in this role and support them within it, allowing them assess to the nutritional services the children in their care so vitally need. More generally, in any emergency setting, organisations need take into account all stakeholders in child nutrition when planning and implementing programmes.

\section{G268(P) ARE ESSENTIAL MEDICINES AVAILABLE, RELIABLE AND AFFORDABLE IN LOW-MIDDLE INCOME COUNTRIES?}

${ }^{1,2}$ TOB Eden, ${ }^{2}$ E Burns, ${ }^{2}$ P Freccero, ${ }^{3} \mathrm{~L}$ Renner. ${ }^{1}$ Academic Unit of Paediatric and Adolescent Oncology, University of Manchester, Manchester, UK; ${ }^{2}$ Programmes Department, World Child Cancer UK, London, UK; ${ }^{3}$ Paediatric Department, Korle Bu Teaching Hospital, Accra, Ghana

\subsection{6/archdischild-2018-rcpch.261}

Background There is increasing anxiety worldwide that the World Health Organisation recommended Essential Medicines may not be universally available,accessible,affordable and of good quality.

Aims/methods We wished to seek the perceptions of 10 lead paediatricians treating children with cancer in 9 low-middle income countries. We used a 17 point semi-structured questionnaire to gather the information. this study involved doctors in Cameroon,Ghana, Malawi,Tanzania,Zambia, Bangladesh,Myanmar, The Philippines,and Colombia. Collectively the centres were seeing overr 2000 new patients annually of whom a median of 65\% (range 5\%-90\%) received 'curative intent' therapy.

Results Only Bangladesh produced any but not all the required cytotoxics. All countries were required to import some or all the medicines.In only 3 countries did the Ministry of Health directly oversee procurement/importation of drugs. In 5 delegated pharmacies or a single hospital was responsible. in Bangladesh there was a free market approach for procurement and importation. Inconsistent supplies were cited by all respondents especially of critical drugs including antibiotics, morphine, 6-mercaptopurine, methotrexate,cytosine, asparaginase, and vincristine. Reasons cited for no-importation were non/or late renewal of licences to import, reluctance to procure low profit or low volume drugs and anxieties regarding importation of opiates. Medicines were imported from a range of countries most commomly from India (7/9) and China (often via India), argentina,Brazil, South Kores, Cyprus and Malaysia. All respondents expressed anxiety about drug quality imported with no International Quality Certification. High rates of treatment refusal/abandonment $(20 \%$ median range $<5 \%-50 \%)$ were most often attributedto non-affordability by parents unless some subsidies were available.

Conclusions From these perceptions there are major obstacles to be overcome to ensure that all children can receive the medicines they need for any chance of cure certainly those living in low-middle income countries. There are challenges at each stage from production to the bedside and only a truly global effort by all interested parties including the WHO and the Pharmaceutical Industry can resolve these issues.

\section{G269(P) INCREASING BURDEN OF THE IMPACT OF CLIMATE CHANGE ON PAEDIATRIC INFECTIOUS DISEASES}

${ }^{1} \mathrm{G}$ Oligbu, ${ }^{2} \mathrm{~A}$ Adepoju. ${ }^{1}$ Paediatric Infectious Diseases Research Group, St George's University of London, UK; ${ }^{2}$ Department of Paediatric and Child Public Health, University College Hospital, Ibadan, Nigeria

\subsection{6/archdischild-2018-rcpch.262}

Introduction Climate change refers to long-term shifts in weather conditions and patterns of extreme weather events, with recent earthquakes, natural disasters, there is epidemic and increase in many paediatric infectious diseases (PID) worldwide, including some newly-circulating ones, such as Zika virus and arbo-viruses in North America and the Caribbean, Ebola and Cholera outbreak in Africa, SIRS in middle east e.t.c. This reflects the combined impacts of rapid demographic, environmental, social, technological and other changes in our ways of living.

Aims We reviewed the available scientific evidences of the impact of recent climate change on PID and also examines the observed and predicted impacts of changes in major climate variables on pathogen, host, and transmission of PID in order to estimate the future burden of infectious diseases on children.

Methods We reviewed published and unpublished literature on climate change and PID. The data were mainly descriptive and we used predictive analytical modelling to assess the future burden.

Findings We observed evidence of associations between climatic conditions and PID in children with different modality of transmission. The responsible agents identified were viruses, bacteria, protozoa and parasites, with various adaptations in the host. Increasing temperature was the most important contributing factor when compared with other natural disasters.

There is a global resurgence of hitherto controlled diseases and the emergence of new ones with redistribution of existing infectious diseases due to an unstable climate. Many of the most common infectious diseases, and particularly those transmitted by vectors, are highly sensitive to climate variation. New and resurgent vector-borne communicable diseases, including arbo-viruses, such as dengue, Zika and Ebola, and malaria are evident widely. Other infectious diseases, such cholera have shown increased outbreaks due to elevated temperature and flooding with the resultant displacement of affected people.

Conclusions Changes in infectious disease transmission patterns are a likely major consequence of climate change. There is need for a better understanding of the causal relationships, in order to predict the future impacts of this on children's health. In addition, paediatrician are likely to see an increasing number of cases due to adverse effects of climate change. An international collaborative effort with an integrated model of care is therefore required.

\section{G270(P) POST-OPERATIVE OUTCOMES OF GAMBIAN CHILDREN AND ADOLESCENTS POST VALVULAR SURGERY FOR RHEUMATIC HEART DISEASE}

${ }^{1,2} \mathrm{~K}$ Martin, ${ }^{1} \mathrm{~K}$ Forrest, ${ }^{1} \mathrm{~L}$ Jaiteh, ${ }^{1} \mathrm{R}$ Maane, ${ }^{1} \mathrm{~S}$ Anderson. ${ }^{1}$ Clinical Services Department, Medical Research Countil Unit The Gambia, Fajara, The Gambia; ${ }^{2}$ Brighton and Sussex University Hospitals NHS Trust, Brighton, UK

10.1136/archdischild-2018-rcpch.263 